\title{
La satisfacción laboral de los servidores públicos en Tungurahua
}

\section{Introducción}

El interés en los últimos años sobre la satisfacción en el trabajo ha incitado a realizar estudios que permitan conocer no sólo el nivel de bienestar de los trabajadores, sino también que ésta sea considerada como una variable en la calidad de vida de los mismos.

La complejidad actual que presenta el entorno de trabajo de los funcionarios ha propiciado que los retos a los que se enfrentan sean cada vez mayores, demandando un personal más moderno, competitivo, con mayor grado de compromiso con la institución $y$, a su vez, que goce de más autonomía en su trabajo.

\section{Importancia de la satisfacción laboral}

El análisis de la satisfacción laboral se ha convertido en un factor clave para alcanzar una adecuada gestión de los recursos humanos dentro de las organizaciones.

En el caso de las instituciones públicas, es preciso adaptarse a los cambios y, así, poder satisfacer de forma general las necesidades de sus trabajadores. Esto significaría un problema en la práctica debido a la rigidez que presenta el sector y a que cada trabajador tiene distintas preferencias según su cultura, educación, edad, entorno y escala de valores.

La presión que puede sufrir un trabajador en la realización de su trabajo no sólo afecta a su rendimiento dentro de la organización a la que pertenece, sino también a nivel personal, familiar y social. Las consecuencias más conocidas son el estrés y el síndrome de quemado, los cuales presentan un riesgo para las organizaciones ya que se traduce en absentismo laboral, rotación y abandono.

En el último censo Poblacional y de Vivienda realizado en Tungurahua (2010) el porcentaje de "Empleados de Estado" es del $8,2 \%$ que corresponde a 19.504 personas respecto al total de la población de la provincia.

Figura 1: Población económicamente activa en Tungurahua Año 2010

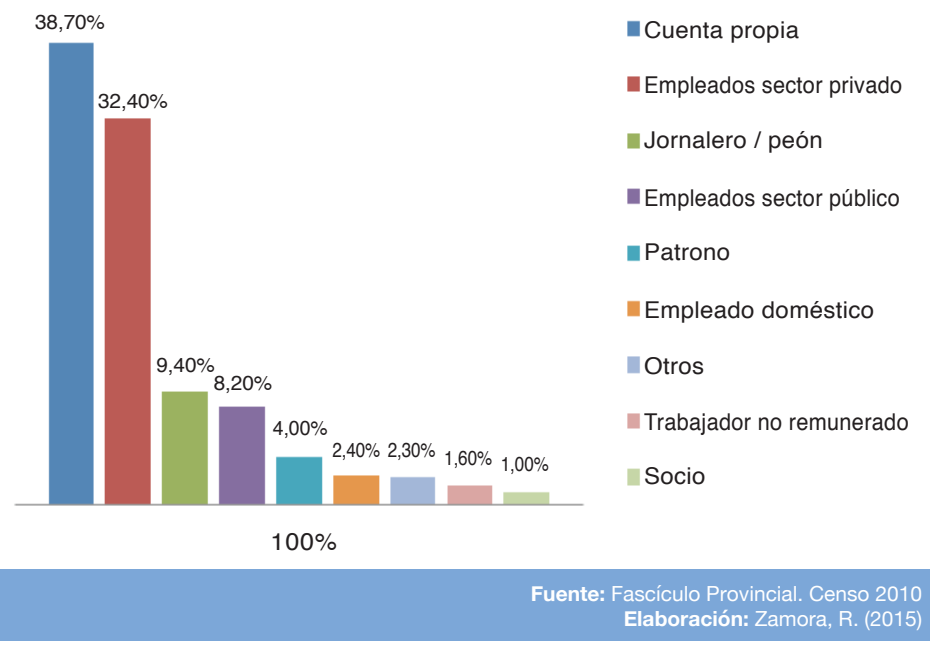

Porcentaje significativamente mayor respecto al año 2001 con un $2,81 \%$ de la población con un total de 12.397 personas.

Figura 2: Porcentaje Población Económicamente Activa en Ecuador Año 2010

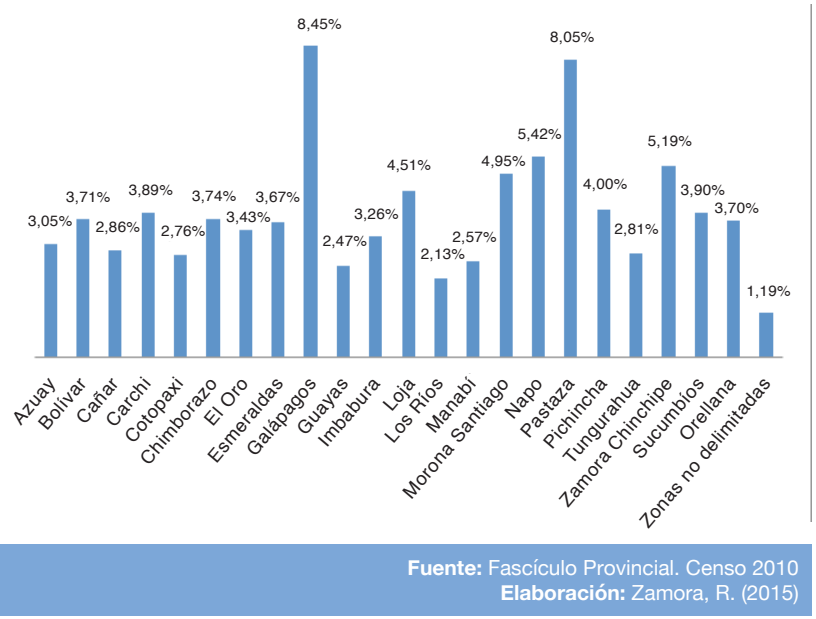

En el sector público resulta difícil motivar a los trabajadores mediante aumentos salariales, reducciones de horarios de trabajo o prestaciones extrasalariales, ya que estas medidas están establecidas por Ley.

A principios de 2015, el Presidente de la República, Economista Rafael Correa, comunicó una reducción salarial de los Funcionarios Públicos del nivel jerárquico superior, la primera en su mandato desde el año 2007. La reducción salarial está comprendida entre un $5 \%$ y un $10 \%$ dependiendo de la categoría del cargo que se desempeñe. Actualmente, la distribución de los servidores públicos según sectores es la siguiente:

Figura 3. Población Económicamente Activa en Ecuador Comparativo entre años 2006 y $2015^{*}$

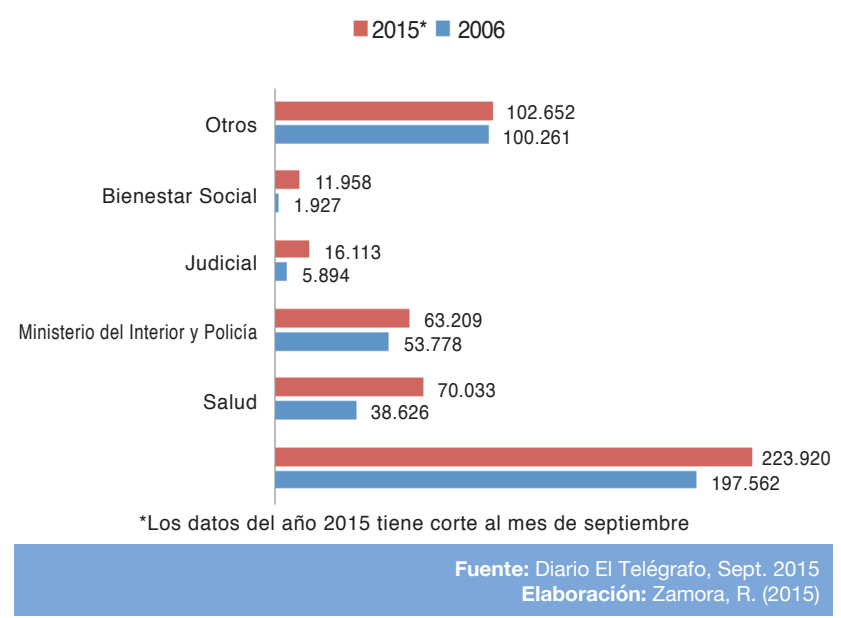


El análisis se realiza entre los años 2006 y 2015, para hacer una comparativa de la variación de número de servidores públicos que ha tenido lugar durante el mandato del Economista Rafael Correa. Como se puede apreciar en la figura 3, el incremento de la cantidad de trabajadores del sector público no es considerable, por lo que el gasto público destinado a pago de sueldos de dichos funcionarios se ha incrementado en el $1.13 \%$ en sector de educación; $6.21 \%$ bienestar social, debido a la creación de políticas que beneficien y protejan a los sectores más vulnerables del país.

\section{Factores psicosociales y sus efectos en el personal}

Los riesgos psicosociales en el trabajo comprenderán todos aquellos factores del contenido laboral y organizacional que actúan como potenciales fuentes de presión, como estresantes o como desencadenantes del estrés laboral (Cox et al., 2005).

Varios autores han realizado estudios sobre el estrés y sus consecuencias en el individuo como: el agotamiento emocional y baja realización personal (Maslasch y Jackson, 1981) o surgimiento de problemas psicofisiológicas o psicosomáticas (Labrador y Crespo, 1993), tales como dolor de cabeza, problemas digestivos, etc. Igualmente han investigado las consecuencias del Burnout o síndrome del quemado (Carlin y Garcés de los Fayos Ruiz, 2010; Cordes y Dougherty, 1993; Figueiredo-Ferraz et al., 2009; Jackson y Manning, 1995; Lozano et al., 2007; Malach, 2002; entre otros), que se conceptualiza como una respuesta al estrés laboral crónico, y por tanto, se relaciona al desgaste profesional que sufre el trabajador.

A nivel psíquico, los trabajadores presentan pérdida de interés por el trabajo, absentismo laboral, conflicto con sus compañeros, entre otros. También se reconoce la necesidad de rediseñar los puestos de trabajo y sistemas de trabajo para aumentar la productividad de las organizaciones y, a su vez, mejorar la calidad de las experiencias de trabajo de los miembros de la organización.

Por ello, en algunos casos, el absentismo es resultado de la incompatibilidad de la jornada laboral con su vida familiar. Es necesario que las organizaciones diseñen nuevos sistemas de modelos de trabajo que se adapten mejor a la situación actual a la que se enfrenta la organización, así, como las capacidades del trabajador.

Todos estos factores tienen consecuencias económicas negativas en las organizaciones, debido a que afecta al nivel de productividad de los trabajadores (Álvarez, 2009).

\section{Reflexiones}

Dentro de una organización, los individuos pueden desarrollar sus actividades laborales en un entorno favorable y conseguir que el trabajador brinde su aporte de sus conocimientos y habilidades; o un entorno desfavorable, donde el trabajador se sentirá desmotivado y que puede llevarle a cambiar de trabajo.
En las últimas décadas se ha realizado un gran esfuerzo en investigar el tema de la satisfacción de los trabajadores. La presión que pueda ejercer el trabajo y la organización sobre el trabajador es fundamental para analizar su nivel de satisfacción en el entorno de trabajo, así, como su motivación en el desempeño de las tareas que le son asignadas al individuo.

Hay que recalcar que la satisfacción que tenga el trabajador influirá en la atención que estos servidores públicos ofrezcan a los ciudadanos que día a día se acercan a las instituciones públicas a realizar sus trámites, así como en la productividad de la organización en general.

\section{Referencias: \\ - Álvarez, P. (2009). Los riesgos psicosociales y su reconocimiento como enferme- dad ocupacional: Consecuencias legales y económicas. Revista Telos, N 11 (3), Pp. $367-385$. \\ - Carlin, M. y Garcés de los Fayos Ruiz, E. (2010): El síndrome de Burnout: Evolución histórica desde el contexto laboral al ámbito deportivo. Anales de Psicología, Vol.} $26, \mathrm{~N}^{\circ} 1$ (enero), Pp. $169-180$

- Cordes, C. y Dougherty, T. (1993): A Review and an Integration of Research on Job Burnout. The Academy of Management Review, Vol. 18, № 4, Oct., 1993, Pp. 621 656.

- Cox, T et al. (2005). Research on Work - related stress. Luxemburgo. Agencia Europea para la seguridad y salud en el trabajo.

- Diario El Telégrafo (2015): http://www.telegrafo.com.ec/politica/item/solo-el-9-de-empleados-es-del-sector-publico.html

- Fascículo Provincial Tungurahua. Resultados de Censo 2010.

- Figueiredo-Ferraz, H. et al. (2009): Prevalencia Del Síndrome de Quemarse por E Trabajo (Burnout) en una muestra de maestros portugueses. Publicado en la Revista Aletheia, Vol. 29, Jan-Jun 2009, Pp. 6 - 15

- Gil-Monte, P. et al. (2006): Relación entre el Síndrome de Quemarse por el trabajo (Burnout) y Síntomas Cardiovasculares: Un Estudio en Técnicos de Prevención de Riesgos Laborales. Revista Interamericana de Psicología, Vol. 40, № 2, Pp. 227 232.

- Hackman, J. R. and Oldham, G. R. (1.980): Work redesign. Massachusetts: Adisson - Wesley Reading.

- Herzberg, F., Mausner, B., Bloch Snyderman, B. (1959): The Motivation to Work. Versión en castellano "La motivación para el trabajo". Wiley International Co.

- Jackson, C. y Manning, M. (1995): Burnout and Health Care Utilization. Journal of Health and Human Services Administration, Vol. 18, No 1, Summer - 1995, Pp. 31 43.

- Kast, F.E. and Rosenzweig, J.E. (1985): Organization and Management: A Systems and contingency approach. McGraw Hill, 4th. Edition.

- Labrador, F. y Crespo, M. (1993): Estrés. Publicado en Madrid, Eudema.

- Lozano, L. et al. (2007): Descripción de los niveles de Burnout en diferentes colectivos profesionales. Aula Abierta 2007, Vol. 36, No 1-2, Pp. $79-88$.

- Malach, A. (2002): The changing psychological at work and employee burnout Journal of Health and Human Services Administration, Vol. 25, № 1 , Summer 2002, Pp. $11-32$.

- Maslach, C. y Jackson, S. (1981): The measurement of experienced burnout Journal of Occupational Behaviour, Vol. 2, Pp. $99-113$.

- McClelland, D.C. (1989): Human Motivation. Traducido al Castellano como Estudio de la Motivación Humana, por Narcea, S.A. de Ediciones, Madrid.

- Rissén, D. (2006): Repetitive and monotonous work among women: Psychophysiological and subjective stress reactions, muscle activity and neck and shoulder pain. Department of Psychology, Stockholm University.

Elaboración:

Lic. Ruth Zamora Sánchez, Ms.C. 